\title{
Resolution of Multi-Party Security Clashes in Social Networking
}

\author{
V.Sathiya Suntharam, Ravi Kumar Chandu, S.Anitha
}

\begin{abstract}
Topics shared through Social Media may also additionally furthermore impact a couple of customer's protection - e.G., images that depict numerous customers, feedback that kingdom numerous clients, sports activities in which numerous clients are invited, and masses of others. The nonappearance of multi-amassing insurance commercial enterprise agency assist in present day-day massive Social Media establishments makes customers not organized to correctly control to whom those items are clearly shared or now not. Computational gadgets that could merge the assurance tendencies of diverse clients proper into a lone tool for and problem can deal with this trouble. The ones strategies want to recall how customers would possibly sincerely go to a comprehension spherical a reaction for the dispute with a selected remaining objective to recommend exercising plans that can be palatable via maximum of the clients stricken by the factor to be shared. Modern-day strategies are both unreasonably bringing up or really preserve in thoughts adjusted strategies for conglomerating safety inclinations. Here we endorse the precept computational device to decide conflicts for multi-celebration safety organisation in Social Media that could follow large situations via displaying the concessions that clients make to perform a response for the disputes. We moreover gift involves fruition of a patron take a look at in which our proposed framework defeated unique present techniques to the quantity how generally each technique facilitated customers direct.
\end{abstract}

Key phrases- Interactive multimedia, privacy, Social networking, Conflicts, Social media, Multi-celebration protection

\section{ADVENT}

A massive range of things which might be moved to on line networking are co-asserted thru awesome clients [5], except surely the consumer that actions the detail is authorized to set its safety settings (i.E., who can get to the element). This is a notable and difficult problem as clients protection tendencies for co-had subjects essentially complication, so utilising the tendencies of champion social occasion perils such subjects being granted to undesired recipients, which can incite coverage encroachment with proper results (e.G., customers losing their vocations, being virtual stalked, and so on.) [2] .Times of factors be part of pictures that depict terrific humans, remarks that usa numerous clients, activities wherein exquisite customers are invited, and so on. Multi-celebration coverage employer is, as such, of earnest significance for clients to because it want to be make sure their protection in Social Media. There can

Revised Manuscript Received on September 10, 2019.

Dr.V.SathiyaSuntharam, Department Of CSE, Professor, CMR Engineering College, Hyderabad, Telangana, India.

(E-mail: sathiya4196@gmail.com)

Ravi Kumar Chandu, Department Of CSE, Associate Professor, CMR Engineering College, Hyderabad, Telangana, India.

(E-mail: chandhuravikumar@cmrec.ac.in)

S.Anitha, Department Of CSE, Asst.Professor, CMR Engineering College, Hyderabad, Telangana, India.

(E-mail: anitharajgk1207@gmail.com) be an ongoing verification that customers all of the time put together [4] cooperatively to carry out a coalition on privateness settings for co-possessed gaining knowledge of in Social media. Specially, clients are outstanding to be generally open to offer agreement to great playing and they are eager to make a few changes to gather at an agreement depending upon the specific conditions [6]. However, gift Social Networking is privateness controls to apprehend this form of situations with the beneficial useful resource of simply the use of the sharing dispositions of the social occasion that transfers a issue, so customers are forced to get making use of precise techniques, for instance, email, SmS, cell telephone calls and so on [1], e.G, Krishna and Madhu may additionally change a few messages to talk approximately whether or no longer or now not or now not they genuinely percentage their picture with Aarush. The problem with that is to accumulate the usage of each one of the conflicts that display up in normal lifestyles is probably time engrossing in mild of the huge quantity of plausible shared gadgets and huge large shape of capacity inspectors to be taken into consideration through the usage of the use of clients. E.G, a solitary consumer in facebook has in more of 160 companions and transfers in greater of 30 pix [3]. An exploration has been top notch as one of the high-quality hole in privateness the board in social networking [6]. The critical problem is to determine the problems that can be cited greater frequently than now not through way of each one of the customers which may be engaged with an object, so customers are pressured to get little as can be expected beneath the times, alongside those lines we're capable of solve multi-celebration privacy conflicts in social media [11] [1]. As we're making use of compromise as a calculation to painting this multi-birthday party privacy conflicts.

\section{RELATED WORKS}

\section{Risks in Social Networks}

Administrators of online social structures [7] are regularly sharing in all likelihood touchy records about clients and their institutions with publicists, software program designers, and data mining experts. Privateness is often secured by way of using anonymization, i.E., expelling names, addresses, and so on. There may be a shape for reading privacy and obscurity in social systems and boom every exceptional re-distinguishing evidence calculation specializing in anonymized social device charts. To delineate its viability on certifiable structures, we show that $33 \%$ of the customers who may be checked to have debts on 
both Twitter, a widely recognized micro strolling a blog manage, and Flickr, an internet photograph sharing net website, can be re-outstanding inside the unknown Twitter chart with nice a $12 \%$ blunder fee.

\section{Disclosure in Social community services}

The multiplication of on-line social systems [8], and the concomitant accumulation of consumer records, provide ascent to fervently noted troubles of privateness, safety, and manipulate. One explicit check is the sharing or open arrival of anonymized information with out coincidentally spilling with the aid of and through recognizable information (PII). Sadly, it's far often tough to find out that diffused measurable strategies, probably using more outdoor records assets, cannot harm obscurity. We hold in thoughts an example of this problem, wherein the item of intrigue is the shape of a social machine, i.E., a diagram portraying clients and their connections. It well-knownshows that anonymizing hub characters may not be top sufficient to keep the tool private the accessibility of hub and connection information from every distinct vicinity, it is associated with the anonymized prepare, has been implemented to redistinguish the anonymized hubs. This is set situations under which this sort of de-anonymization technique is possible.

\section{Multi-agent structures}

Professionals typically typify their principals man or woman information inclinations, which can be uncovered [8] to particular operators for the duration of operator collaborations, growing a capability loss of privateness. Right here we propose self-publicity important management systems for operators to pick out out whether or not or not or not unveiling individual data ascribes to distinct professionals is right enough or now not. Moreover, we likewise endorse at ease operator frameworks to make sure the information that specialists pick out out to discover from undesired receives to.

\section{Analysis of relevance and redundancy}

In exemplary directed gaining knowledge of, one is given a schooling set of marked steady-duration embody vectors (occurrences). An incidence is typically portrayed as a mission of competencies $\mathrm{f}=(\mathrm{f} 1, \ldots, \mathrm{fN})$ to pretty some highlights $\mathrm{F}=(\mathrm{F} 1, \ldots, \mathrm{FN})$ and brought into consideration certainly one of 1 ability training $\mathrm{c} 1, \ldots, \mathrm{cl}$ to the elegance mark $\mathrm{C}$. The assignment is to prompt a precept that precisely predicts the marks of novel occurrences. The mastering of the classifier is inalienably controlled through the usage of the detail esteems. In greater highlights must deliver moreover segregating pressure, however nearly speaking, with a confined degree of getting equipped information, characteristic desire has been a functioning and inexperienced place of modern-day paintings for decade sin actual, AI machine and insights. It has confirmed in each hypothesis and workout successful in improving reading skillability, growing prescient exactness, and reducing multifaceted nature of scholarly. Supply G a threat to be some subset of $\mathrm{F}$ and $\mathrm{fG}$ be the in reality certainly well worth vector of $\mathrm{G}$. At the same time as all is said in completed, the purpose of highlight dedication may be formalized as choosing a base subset $G$ to such an amount $G=f G$ ) is identical or as near as potential $F=f$ ), in which $G$
$=\mathrm{fG})$ is the opportunity flow into of severa training given the element $\mathrm{F}=\mathrm{f}$ ) is the number one conveyance given the element esteems in F. We preserve in thoughts this kind of base subset an tremendous subset.

\section{Warfare decision in Social Media}

Consumer inquiries are commonly too quick to even preserve in mind describing the facts want precisely. Severa large phrases can be missing from the inquiry, prompting a awful inclusion of the pertinent facts. To attend to this problem, query extension has been usually implemented [9] [10]. Among every one of the methodologies, pseudopertinence criticism (PRF) abusing the recovery give up stop quit end result has been the first-class [10]. The important supposition of PRF is that the pinnacle-located documents within the critical recovery give up cease end result contain numerous precious phrases that might help separate pertinent evaluations from unimportant ones. All in all, the extension phrases are removed every as indicated via the term conveyances inside the complaint statistics or as in step with the correlation the various time period dispersions inside the enter statistics and in the whole report accumulation. Some extra necessities were proposed. For instance, idf is commonly implemented in vector place version [9]. Question length has been taken into consideration in [13] for the weighting of improvement phrases. A few phonetic highlights have been attempted [12] in. Be that as it may, few examinations have legitimately inspected whether or not or now not or no longer the extension terms removed from pseudo-enter critiques with the aid of manner of the present day strategies can virtually manual recovery. Even as all is stated in performed, one grow to be worried uniquely with the global impact of plenty of extension terms on the recuperation viability. A key inquiry regularly left out at is whether the extension terms eliminated are sincerely identified with the question and are valuable for IR. Reality be suggested, as we're able to seem in this exploration paintings, the supposition that maximum improvement phrases separated from the input documents are treasured does not keep, however whilst the global recuperation viability may be advanced. Some of the separated terms, a non-immaterial element is every random to the inquiry or is hurtful, in choice to accommodating, to recuperation viability. So a essential inquiry is: how can also we better pick precious extension phrases from pseudo-enter documents. This method is not much like the current ones, that may normally be considered as a solo gaining knowledge of. SVM [3][14] might be applied for term characterization, which makes use of now not truly the term conveyance necessities as in beyond investigations, but further some greater requirements, for example, time period closeness. 


\section{Gadget version}

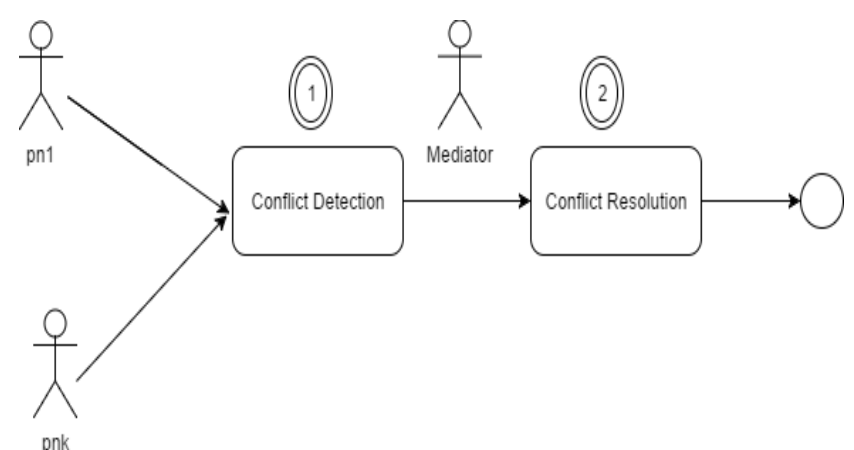

Fig 1: device version form

because of the truth the mediator goes approximately because of the reality the man or woman privacy strategies of the extremely good amount of clients for the issue and conflicts determined. Basically the character privateness conflicts define that picks for the same cause consumer. This conflicts are located in a element that isn't shared at gift. Mediator kills to decide this trouble for conflicts decided. Presently consumer might be to renowned through thinking about how man or woman privacy tendencies are influencing unique consumer and clashing a chunk of writing for the customers.

\section{PROPOSED SCHEME}

Modules

This studies artwork consists of six modules:

1. Man or woman Interface format

2. Posting image

3. Photograph Encryption

4. Picture Request

5. Photo reaction

6. Image Inbox

\section{Client Interface layout}

For interfacing with the server client want to offer their records like username and mystery word then surely patron gets related to the server. At the off risk that the purchaser as of now exists straightforwardly can login into the server else purchaser need to sign on their subtleties, for instance, username, mystery phrase and e-mail identity, into the server. Server will make the file for the entire patron to hold up switch and down load rate. Call is probably set as customer identity. Logging is commonly used to go into a selected net web page.

\section{Posting photograph}

on this module the patron can put up a image on their timetable and he/she can be capable of put up a statement for the pics published at the route of activities.

\section{Photograph Encryption}

on this module the image transferred through the consumer can certainly view the picture incredible clients, for example, partners and extremely good humans aren't organized to look the image because of the truth the photo has been as of now unscrambled itself alongside the ones strains giving safety to the purchaser.

\section{Photo Request}

on this module as client can not see the neighbor's pictures that have been shared through manner of the man or woman on their direction of activities. So the neighbor customer can prepared to deliver a solicitation to the proprietor of the image.

\section{Photograph reaction}

in this module the clients who want the photograph that has call for inside the solicitation vicinity of the proprietor in the event that he/she widely identified the solicitation then the neighbor can see the picture.

\section{Picture Inbox}

in this module if the owner of the picture recognizes the solicitation the neighbor no longer actually organized to look the photograph he/she will be able to prepared to download and the photograph will shared to personal inbox of the neighbor.

\section{OUTCOMES \& RESULTS}

The snap shots and files transferred inside the social media are open to all the social media customers wherein it want a trouble to tie down the common inclination to each one of the records within the social media. To understand this encoding the information and pix transferring to the consumer will create pertinent association which makes the document and snap shots more established within the social media. The competition is fathomed with the beneficial aid of choosing the interest desired through manner of the customer that transfers the detail. To avoid conflicts that occur in social media we are the use of compromise calculation. We use java shape to execute the above technique to illustrate the running of the framework.

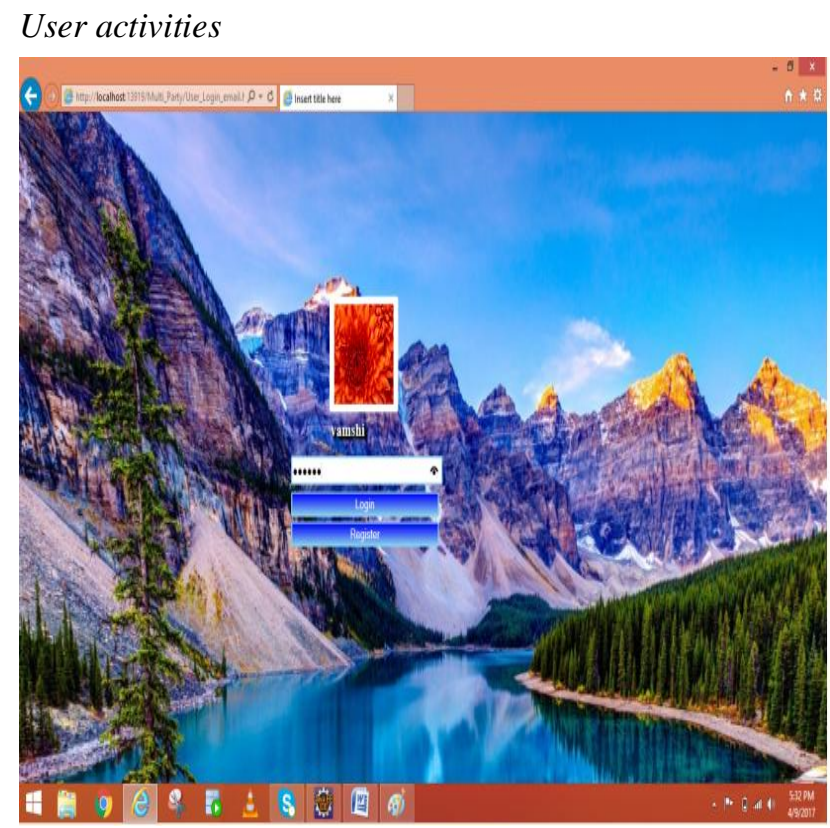

Fig2. Login page 


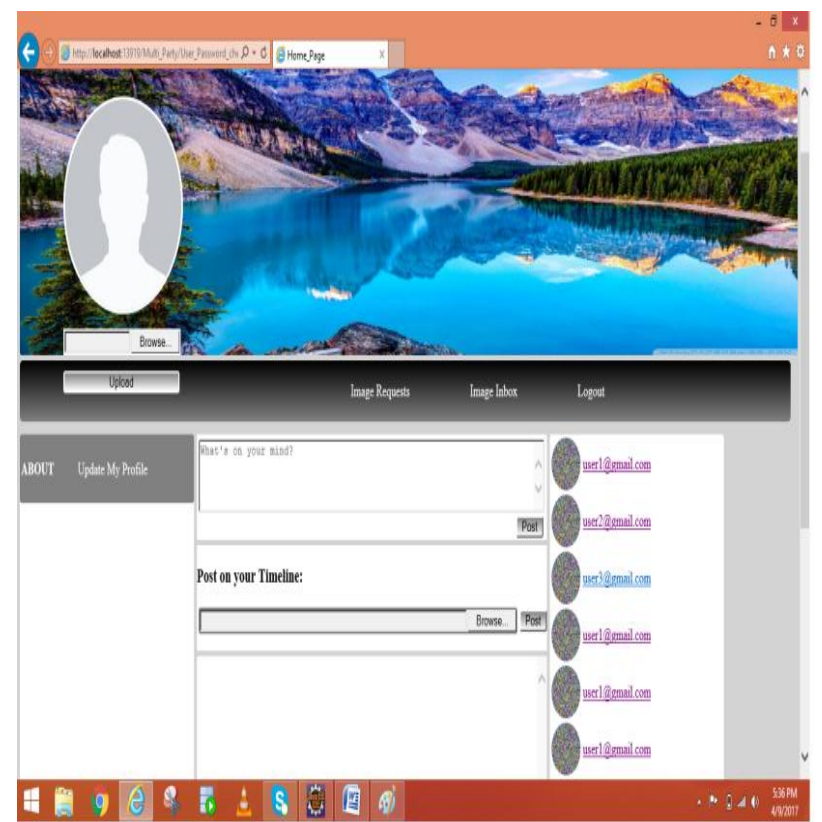

Fig3. Home page

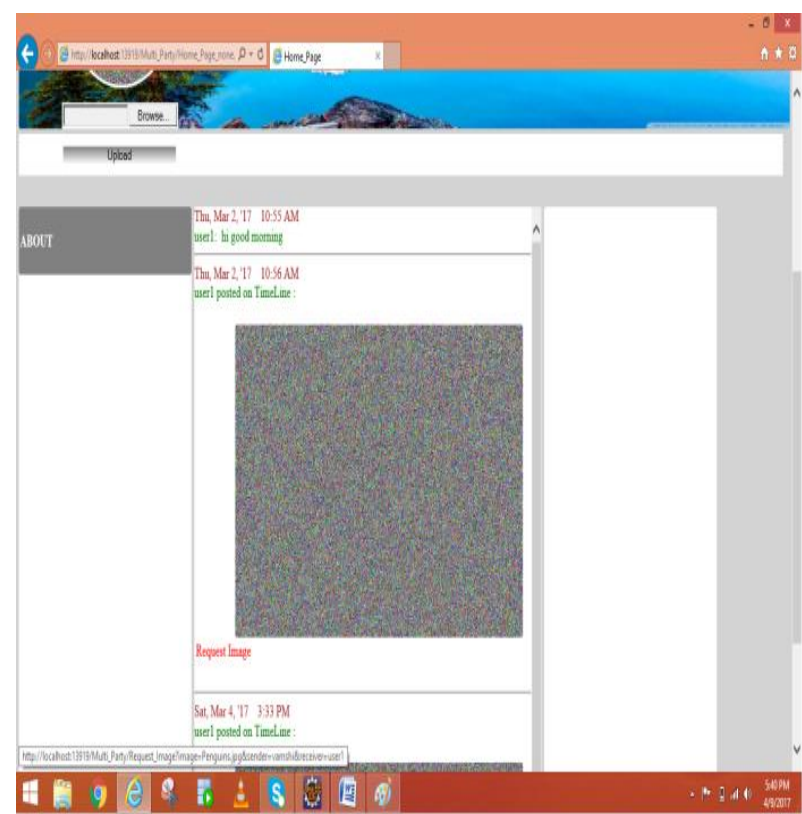

Fig4. Encrypt image

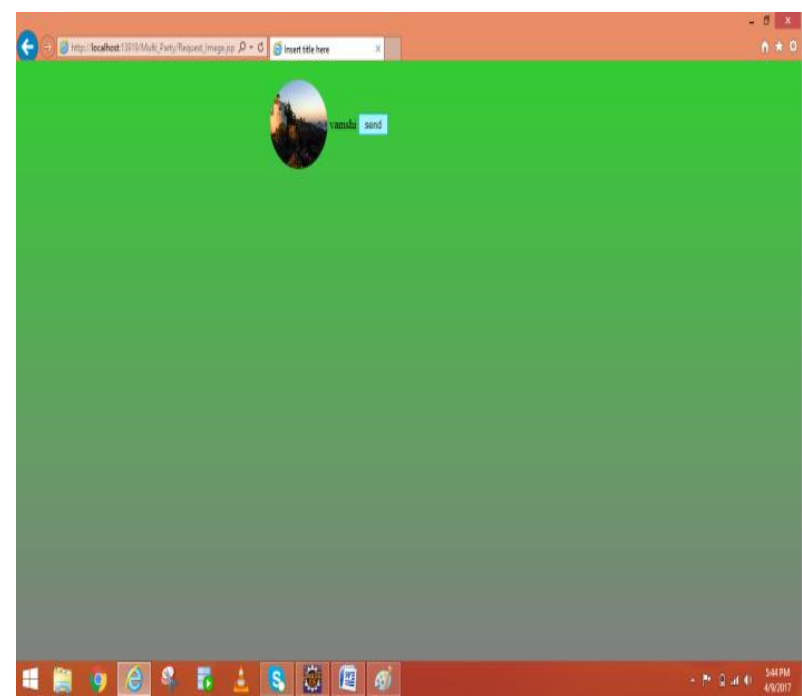

Fig5. Request image

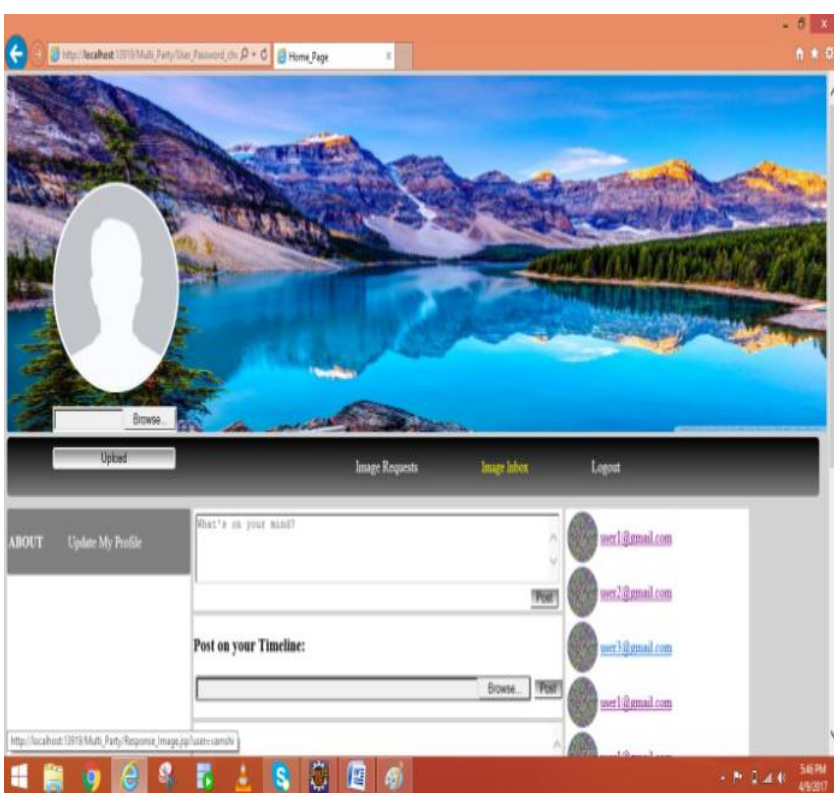

Fig6. Inbox image

\section{V.STOP}

The framework proposed through us, fights decrease again the issues regarded most of the changed gatherings and recognizes and determine coverage conflicts in social media to provide a middle floor to the clients. The framework likewise is strong in adjusting the dispute assure to the precise situations.

\section{CONCLUSIONS}

We proposed the critical computational device to decide conflicts for multi-party guarantee company business enterprise in Social Media that can trade in accordance with unique situations with the useful resource of displaying the concessions that customers make to perform a reaction for the disputes. We in like way gift takes area of a customer test wherein our proposed framework outmaneuvered specific gift structures to the quantity how as often as possible each tool facilitated customers lead.

\section{REFERENCES}

1. A. Besmer and H. Richter Lipford, "shifting beyond untagging: photo privacy in a tagged global," in ACM CHI, 2010, pp. 1563-1572.

2. okay. Thomas, C. Grier, and D. M. Nicol, "unfriendly: Multi-party privacy risks in social networks," in privateness improving technology. Springer, 2010, pp. 236-252. [3] A. Lampinen, V. Lehtinen, A Lehmuskallio, and S. Tamminen

3. facebook NewsRoom, "one thousand million- key metrics," http://newsroom.Fb.Com/down loadmedia/4227, Retr. 26/06/2013.

4. A. Squicciarini, M. Shehab, and F. Percent, "Collective privacy manipulate in social networks," in WWW. ACM, 2009, pp. 521-530.

5. net.Org, "A interest on performance," http://net.Org/efficiencypaper, Retr. 09/2014.

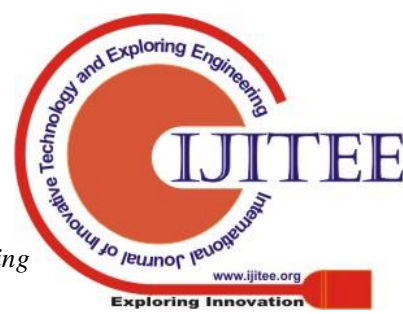


6. P. Wisniewski, H. Lipford, and D. Wilson, "fighting for my location: Coping mechanisms for sns boundary law," in Proc. CHI. ACM, 2012, pp. 609-618.

7. H. Hu, G. Ahn, and J. Jorgensen, "Multiparty get right of entry to manipulate for online social networks: model and mechanisms," IEEE TKDE, 2013.

8. A. Lampinen, V. Lehtinen, A. Lehmuskallio, and S. Tamminen, "We're in it collectively: interpersonal manage of disclosure in social network offerings," in Proc. CHI. ACM, 2011, pp. 3217-3226.

9. P. Fong, "dating-primarily based definitely really genuinely get access to manipulate: protection model and coverage language," in Procs. ACM CODASPY. ACM, 2011, pp. 191-202.

10. M. Sleeper, R. Balebako, S. Das, A. L. McConahy, J. Wiese, and L. F. Cranor, "The publish that wasn't: exploring self-censorship on $\mathrm{fb}$," in complaints of the 2013 convention on pc supported cooperative artwork. ACM, 2013, pp. 793-802.

11. J. M. Such, Natalia Criado "Resolving Multi-celebration privateness Conflicts in Social Media"

12. J. M. Such, A. Espinosa, A. Garcia-Fornes, and C. Sierra, "Self disclosure desire making based completely mostly on intimacy and privacy," statistics Sciences, vol. 211, pp. Ninety three-111, 2012.

13. J. M. Such, A. Espinosa, and A. Garc'ia-Fornes, "A survey of privacy in multi-agent structures," The information Engineering assessment, vol. 29, no. 03, pp. 314-344, 2014.

14. [14] G. Danezis, "Inferring privateness guidelines for social networking offerings," in proceedings of the second ACM workshop on safety and artificial intelligence. ACM, 2009, pp. 5-10. 